\title{
Precise Matching of Semantic Web Services*
}

\author{
Yonglei Yao, Sen Su, and Fangchun Yang \\ State Key Laboratory of Networking \& Switching Technology, \\ Beijing University of Posts \& Telecommunications (BUPT) \\ 187\# 10 Xi Tu Cheng Rd., Beijing 100876, China \\ \{yaoyl, susen, fcyang\} abupt.edu.cn
}

\begin{abstract}
Matchmaking is an important aspect of the Web Services interactions, which enables a service requester to locate the most suitable counterpart. However, current service matching algorithms operate on service advertisements, which don't contain enough information to select a service instance that the consumer can immediately interact with. In this paper, we propose a precise matching algorithm, based on WS-Agreement and OWL-S, to deal with this challenge. We show how to combine WS-Agreement with OWL$\mathrm{S}$ to obtain an agreement-style service description, and how the degree of match between two descriptions of this style is calculated ${ }^{1}$.
\end{abstract}

\section{Introduction}

In recent years, more and more Web Services are becoming available on the Internet, enabling customer to interact with a great number of potential counterparts. On the other hand, this also makes a matchmaking process necessary to locate the most suitable one.

In current matchmaking approaches, the matching algorithms operate on service advertisements and queries [3, 4, 5]. Unfortunately, quality of service and other guarantees cannot simply be advertised. As a result, the matchmaking process can only find a set of services which may possibly meet one's requirements, leaving the task of selecting the most suitable counterpart to the customer.

Against this background, this paper develops a matching algorithm that operates on agreement-style service descriptions, to help a customer to select a specific service that can indeed meets all its requirements. This work advances the state of the art in the following ways. Firstly, it's a novel way to calculate the degree of match between services based on detailed service descriptions which combine WSAgreement [1] and OWL-S [2]. Secondly, this work develops a matching algorithm which can provide a precise and quantified degree of match between a service requested and a service offered, which in turn can serve as a sound criteria for service selection.

* The work presented in this paper was Supported by 973 program of China(2003CB314806), the National Natural Science Foundation project of China(90204007), the program for Changjiang Scholars and Innovative Research Team in University (PCSIRT), and National Natural Science Funds for Distinguished Young Scholar(60125101). 


\section{Matching Algorithm}

In order to select a specific service instance, a service consumer will continuously exchange proposals described in WS-Agreement, in which an OWL-S profile is embedded as service description terms to describe the functionalities provided or required, with potential service providers. Our matching algorithm operates on such agreement-style service descriptions to facilitate the service selection process. Our work presents several matching degree assessment methods as follows:

\subsection{Concept Matching Degree}

Firstly, we show how to calculate the degree of match between two concepts defined in ontologies. We differentiate between four degrees of concept match in terms of the subsumption relationships, and quantify each degree, as shown following:

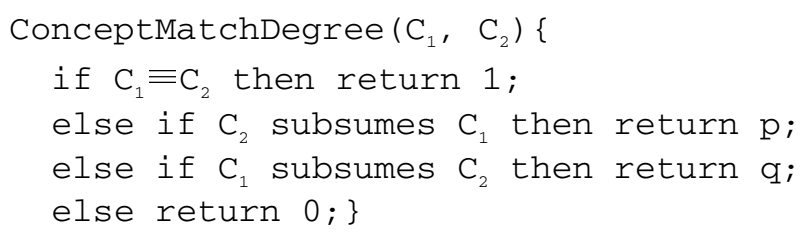

Where $\mathrm{C}_{1}$ and $\mathrm{C}_{2}$ denote two concepts defined in some ontologies, and $\mathrm{p}, \mathrm{q} \in(0,1)$ and $\mathrm{p}>\mathrm{q}$.

\subsection{Input Matching Degree}

For input matching, we want to show how well the inputs and preconditions required by service providers are satisfied by service consumers.

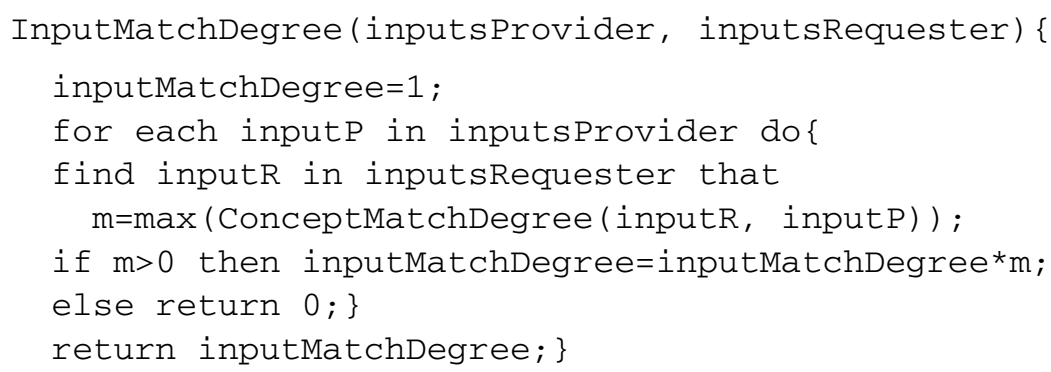

\subsection{Output Matching Degree}

For output matching, we decide how well the outputs and effects required by service consumers are satisfied by those of service providers, as shown following:

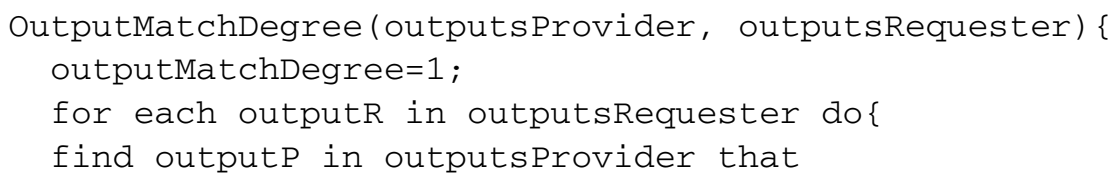




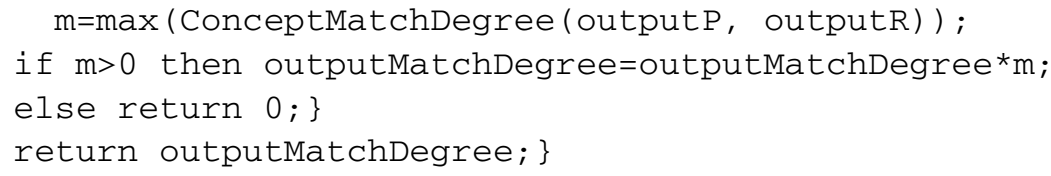

\subsection{Guarantee Term Matching Degree}

Besides functionalities, a service consumer also demands an assurance with respect to the non-functional attributes from the service provider, such as price, delivery time, quality of service, etc. The assurance is described by the guarantee terms of the WSAgreement document.

Let $\mathrm{t} \in\left\{\mathrm{t}_{1}, \mathrm{t}_{2}, \ldots \ldots, \mathrm{t}_{\mathrm{n}}\right\}$ represent the guarantee terms demanded by the service consumer, and $\mathrm{D}_{\mathrm{i}}=\left[\min _{\mathrm{i}}, \max _{\mathrm{i}}\right]$ denotes the intervals of values for quantitative term $\mathrm{t}_{\mathrm{i}}$ acceptable for the service consumer. Values for qualitative issues, on the other hand, are defined over a fully ordered domain, i.e., $D_{i}=<q_{1}, q_{2}, \ldots \ldots, q_{m}>$.

For each term $t_{i}$, the service consumer has a scoring function $V_{i}: D_{i} \rightarrow[0,1]$ that gives a score to a value of term $t_{i}$ in the range of its acceptable values. Based on the above model, we can calculate the degree of match between guarantee terms.

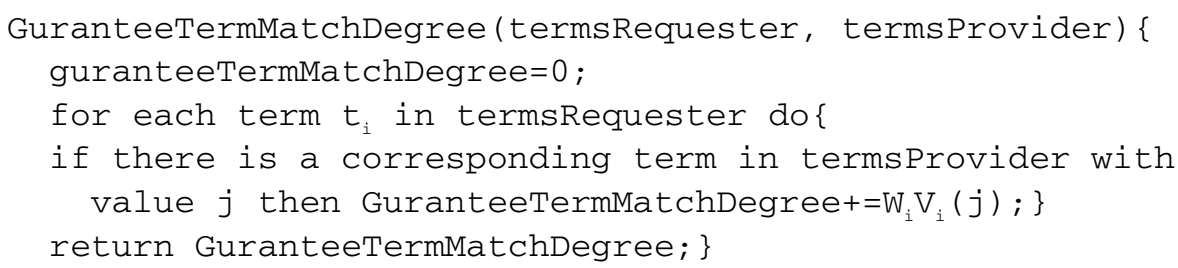

Where $\mathrm{W}_{\mathrm{i}}$ denotes the relative importance that the service consumer assigns to term $\mathrm{V}_{\mathrm{i}}$, The weights of all terms are normalized, i.e., $\sum_{1 \leq i \leq n} W_{i}=1$.

\subsection{Global Matching Degree}

Based on the matching degrees between input parameters, output parameters and guarantee terms, we can determine the degree of match between two agreement-style service descriptions as:

$$
\begin{aligned}
& \text { M atchDegree }=W_{i} \times \text { inputM atchDegree }+W_{0} \times \text { outputM atchDegree } \\
& +\mathrm{W}_{\mathrm{g}} \times \mathrm{Guarantee} \mathrm{T} \text { erm M atch Degree }
\end{aligned}
$$

Where $\mathrm{W}_{\mathrm{i}}, \mathrm{W}_{\mathrm{o}}$ and $\mathrm{W}_{\mathrm{g}}$ represent the weights that the service consumer assigns to input match, output match and guarantee term match respectively.

\section{Conclusion and Future Work}

In this paper, we propose an algorithm for calculating the degree of match between two services based on the agreement-style service descriptions. Given the algorithm we develop, a service consumer can select a specific service which indeed has the ability to meet her requirements. 
Future work includes implementing the matching algorithm, applying this technique to various applications, and as the last step, investigating the effectiveness of the algorithm with real world use cases.

\section{References}

[1] A. Andrieux, K. Czajkowski, A. Dan, K. Keahey, H. Ludwig, J. Pruyne, J. Rofrano, S. Tuecke, and M. Xu, Web Services Agreement Specification (WS-Agreement): Global Grid Forum, May 2004.

[2] D. Martin, M. Burstein, J. Hobbs, O. Lassila, D. McDermott, S. Mcllraith, S. Narayanan, M. Paolucci, B. Parsia, T. Payne, E. Sirin, N. Srinivasan, and K. Sycara. Owl-s: Semantic markup for web services, http://www.daml.org/services/owl-s/1.1/overview/, 2004.

[3] J. Hau, W. Lee, J. Darlington, A Semantic Similarity Measure for Semantic Web Services, In Proceedings of the fourteenth International World Wide Web Conference (WWW 2005), Chiba, Japan, May 10-14, 2005

[4] L. Li and I. Horrocks, "A software framework for matchmaking based on semantic web technology", In Proceedings of the Twelfth International World Wide Web Conference (WWW 2003), pages 331-339, ACM, 2003.

[5] M. Paolucci, T. Kawamura, T.R. Payne, and K. Sycara., "Semantic matching of web services capabilities", In Proceedings of the 1st International Semantic Web Conference (ISWC), pages 333-348, Sardinia, Italia, June 2002. 